\title{
La práctica pedagógica, un escenario de emociones, reflexiones e investigación desde el diario de campo
}

\section{Pedagogical practice, a scenario of emotions, reflections and research from the field diary}

DOI: $10.46932 /$ sfjdv2n2-164

Received in: March 1st, 2021

Accepted in: May 30th, 2021

\section{Aida Astrid Obando Rodríguez}

Magister en Educación. Estudiante de Doctorado en Ciencias de la Educación. RUDECOLOMBIA. Universidad del Tolima

Address: Cra 4B No. 32 A 17 Barrio la Francia. Ibagué-Tolima-Colombia

E-mail: aaobandor@ut.edu.co

\section{RESUMEN}

El proyecto fue desarrollado en la Escuela Normal Superior de Ibagué, con estudiantes del grado $13^{\circ}$ del Programa de Formación Complementaria, quienes se educan para desarrollar procesos pedagógicos en el nivel de Preescolar y de Básica Primaria, según decreto 4790, MEN1 (2008), así como de educación inicial según adición en el decreto 1236, MEN (2020). El objetivo fue interpretar, desde de diarios de campo, las emociones emergentes de la práctica pedagógica, de las cuales poco se reflexiona e investiga en los planes de formación inicial de maestros, instituidos principalmente, en teorías sobre enseñanza y aprendizaje. Lo anterior, es fundamento de mi interés investigativo sobre políticas y prácticas educativas que promueven cultura investigativa para la formación de maestros normalistas superiores, en el Doctorado en Ciencias de la Educación, que actualmente curso con Rudecolombia.

El propósito de la investigación exigió la sistematización, el análisis y la interpretación de 60 diarios de campo de los maestros en formación, en los cuales no solo se narraron las experiencias pedagógicas; sino que se describieron las emociones emergentes en las intervenciones con los niños. Para Porlán (2000) el diario de campo “... es una guía para la reflexión sobre la práctica, favoreciendo la toma de conciencia del profesor sobre su proceso de evolución y sobre sus modelos de referencia" (p,23), acción metacognitiva que se convierte en un ejercicio de investigación educativa permanente.

Los resultados develaron que, las acciones desarrolladas en la práctica pedagógica generan emociones multivalentes que conducen a procesos de autorreflexión, crítica e investigación sobre la misma. Como sujetos sensibles, los maestros no son ajenos a las emociones, que en muchos momentos resultan contradictorias y que convergen en tensiones que desestiman el capital cognitivo y actitudinal del futuro profesional educativo (Bedacarratx,2012) y que limitan el mejoramiento continuo sobre cómo enseñar, cómo comprender las necesidades e intereses de los educandos y del mundo mismo, entre otras cuestiones que se instauran como objetos de investigación educativa, tarea sustancial de los maestros.

Palabras clave: diario de campo, emociones, investigación, práctica pedagógica.

\section{ABSTRACT}

The project was developed at the Normal Superior School of Ibague, with students from the 13th grade of the Complementary Training Program, who are educated to develop pedagogical processes at the pre-

\footnotetext{
${ }^{1}$ MEN. Ministerio de Educación Nacional de Colombia. Máximo órgano rector de los procesos y políticas educativas en el País.
} 
school and primary level, according to decree 4790, MEN (2008), as well as initial education according to the addition in decree 1236, MEN (2020).

The objective was to interpret, through field journals, the emerging emotions of pedagogical practice, which little thought and research into the initial teaching training plans, instituted mainly, in theories of teaching and learning. The foregoing is the basis of my research interest in educational policies and practices that promote research culture for the training of teachers in higher education, in the Doctorate in Education Sciences, which I am currently studying with Rudecolombia.

The purpose of the research required the systematization, analysis and interpretation of 60 field journals of teachers in training, in which not only the pedagogical experiences were narrated; but the emerging emotions in interventions with children were described. For Porlán (2000), the field diary "it is a guide for reflection on practice, promoting the teacher's awareness of its evolution process and its reference models" (p,23), metacognitive action that becomes an ongoing educational research exercise.

The results revealed that the actions developed in the pedagogical practice generate multivalent emotions that lead to processes of self-reflection, criticism and research about it. As sensitive subjects, teachers are not oblivious to emotions, which at many times are contradictory and converge in tensions that dismiss the cognitive and attitudinal capital of the future educational professional. (Bedacarratz, 2012); and that limit the continuous improvement on how to teach, how to understand the needs and interests of learners and the world itself, among other issues that are established as objects of educational research, substantial teachers' task.

Keywords: emotions, pedagogical diary, pedagogical practice, research.

\section{INTRODUCCIÓN}

La formación de maestros ha sido históricamente un compromiso social, político y ético de gran valor, debido a que a los docentes se les ha delegado la responsabilidad de educar a las presentes y futuras generaciones que sustentan la sociedad. Para Colombia además de las facultades de educación, las Normales Superiores, asumen el compromiso de formar con alta calidad maestros para los niveles de preescolar y básica primaria sustentadas en programas que contemplan saberes en el campo disciplinar, pedagógico y socio humanístico. Normativamente, el decreto 4790, emanado por el Ministerio de Educación Nacional, (2008) “establece las condiciones básicas para la organización y el funcionamiento del programa de formación complementaria de educadores para el nivel de preescolar y el ciclo de básica primaria que puede ofrecer una escuela normal superior" (p.1). Dentro de estas condiciones, la referida a las prácticas docentes en el $\mathrm{PFC}^{2}$, es eje articulador de los 12 restantes, pues sin la intervención del docente en el aula y en las demás actividades institucionales sería más complejo comprender la teoría que sobre educación dirigen los diseños curriculares de los programas de formación de maestros. Así mismo, en el año 2015, el MEN define los cuatro ejes vertebradores para la misma misión en las ENS ${ }^{3}$ : evaluación,

\footnotetext{
${ }^{2}$ PFC. Programa de Formación Complementaria. Corresponde a los grados $12^{\circ}$ y $13^{\circ}$, cursados por los estudiantes que quieren ser normalistas superiores para ejercer la docencia en Preescolar y Básica Primaria.

${ }^{3}$ ENS. Refiere a las Escuelas Normales Superiores en Colombia, responsables de formar maestros idóneos para los niveles de Preescolar y Básica Primaria.
} 
proyección social, formación e investigación, los dos últimos sustentos de un maestro crítico-reflexivo. Este recorrido normativo, se confirma en el decreto 1236 de 2020, a través del cual el MEN, reincide en el reconocimiento pedagógico y social de las ENS para la formación de maestros y da apertura a que estas instituciones puedan suscribir convenios con otras instituciones de educación superior, para el desarrollo de proyectos colaborativos de investigación y la producción de saberes.

Por su parte, los maestros que se forman en la Escuela Normal Superior de Ibagué, desarrollan procesos de práctica pedagógica de manera permanente, a lo largo del programa definido para tal fin y los sustentan en valores institucionales (2011) como el compromiso, la idoneidad, la honestidad y el respeto. Estos últimos le dan el carácter humanizante a la formación y se constituyen en elementos que dan el valor ético el quehacer docente. Sin embargo, la existencia de estos valores no condiciona la óptima calidad de la formación docente, pues el maestro en formación ${ }^{4}$, en el ejercicio de su autonomía y autorreconocimiento debe reflexionar permanentemente sobre su práctica pedagógica para evitar caer en posibles contradicciones como lo plantea Massi (2008). De manera recurrente, estas reflexiones se construyen en relación al alcance de las metas propuestas en el campo de lo metodológico o de lo normativo sobre lo cual se erige la práctica; pocas veces el estado emocional del practicante es analizado e investigado desde su experiencia, desde sus interacciones o desde sus producciones escriturales. La formación del maestro, ha estado más bien observada y evaluada a la luz de teorías pedagógicas que dan el sustento profesional a la docencia, pero no se ha profundizado en el ser humano que encarna cada maestro. Para Pekrun (2005) en el contexto educativo los estados anímicos sean positivos o negativos pueden afectar el desarrollo de actividades metacognitivas, el interés por las mismas y el esfuerzo por alcanzar resultados coherentes con la tarea asignada.

En la $\mathrm{ENSI}^{5}$, el proceso de análisis docente se ha promovido a partir de la escritura reflexiva, sistemática y permanente del diario de campo; a través de este se evidencia la praxis sobre la acción pedagógica del maestro en formación. Las narraciones sobre hechos puntuales; las descripciones de lugares, situaciones, intervenciones y sobre todo de las emociones han permitido lentamente afianzar el carácter crítico del normalista superior frente a la docencia, frente a su compromiso personal de formación y frente a su compromiso social transformativo. Se trata pues, desde la reflexión pedagógica, constituir una praxis pedagógica que forme maestros sensibles a las necesidades y emociones de otros, pero también sensibles a sus propias necesidades y emociones. Estas mueven la acción docente, eleva a niveles de exigencia educativa los procesos de las instituciones formadoras de maestros y prospectivamente abre caminos para desarrollar procesos investigativos en el campo de la educación, fruto de la reflexión sobre

\footnotetext{
${ }^{4}$ Maestro en Formación, es el estudiante de los grados 12ㅇ y 13ㅇqu que estudia en la ENS para ser maestro de preescolar y básica primaria. Por sus intervenciones en el aula también es denominado practicante.

${ }^{5}$ ENSI. Escuela Normal Superior de Ibagué.
} 
la acción pedagógica y del liderazgo de los docentes, como plantea Domingo(2013) "Basar la formación en la reflexión propicia docentes autónomos, que promuevan una continua renovación personal, actores creativos a nivel didáctico y no estrictos ejecutores de currículos prediseñados”(p.163).

\section{OBJETIVO}

Interpretar, a través de diarios de campo, las emociones emergentes de la práctica pedagógica como un hecho de interés investigativo permanente.

\section{MARCO TEÓRICO}

Abordar el tema de las emociones en el contexto de la práctica pedagógica, es un asunto importante porque permite principalmente, el auto reconocimiento del maestro como sujeto senti-pensante y no solo como profesional de la enseñanza; en segundo lugar, porque registrar dichas emociones en un diario de campo, posibilita hacer praxis sobre las intervenciones e interacciones del maestro en su quehacer pedagógico, lo cual se constituye en un acto investigativo permanente y connatural del maestro, con lo cual se aspira a transformar los procesos educativos, en procura de mejores desarrollos en el acto de enseñanza y de aprendizaje. Para el caso particular se tienen en cuenta, entre otros, los siguientes referentes teóricos:

Emociones en el contexto educativo: (Pekrun,2005) en el contexto educativo los estados anímicos sean positivos o negativos pueden afectar el desarrollo de actividades metacognitivas, el interés por las mismas y el esfuerzo por alcanzar resultados coherentes con la tarea asignada.

Investigar sobre emociones: Goetz, Perun, Hall y Haag, 2006, citados por (Paolini,2013), consideran relevante el tema porque el bienestar interior del educando está permeado por sus emociones y las experiencias a que estas converjan; así mismo, las emociones son determinantes en la calidad de los aprendizajes y de los logros que se obtengan. Con respecto a la relación dialógica y académica entre el docente y estudiante, esta también está mediada por las emociones que fluyen en dicha interacción. Finalmente, investigar sobre las “emociones académicas”, genera mejores oportunidades de intervención y promoción de emociones adaptativas en los estudiantes.

El diario de campo: (Porlán, 2000), afirma que El diario “... es una guía para la reflexión sobre la práctica, favoreciendo la toma de conciencia del profesor sobre su proceso de evolución y sobre sus modelos de referencia" (p.23). De esta manera, escribir el diario de campo se convierte más allá de un ejercicio escritural, en una práctica que promueve procesos de mejoramiento pedagógico y personal.

\section{METODOLOGÍA}


La investigación fue de carácter cualitativo y se situó en el paradigma hermenéutico, para la comprensión de las emociones develadas en los discursos de los maestros en formación. Para el desarrollo del proceso metodológico se asumió el diseño cualitativo- narrativo (Hernández,2014), a través de la lectura e interpretación de 60 diarios de campo concedidos por los estudiantes de grado $13^{\circ}$ del Programa de Formación Complementaria durante los años 2018 y 2019. La información encontrada allí fue tratada con confidencialidad y fidelidad de las voces de los escritores, sin perjudicar su identidad, pero si dando un alto grado de veracidad. Lo anterior con el fin de ser coherentes con la ética de la investigación (Hirsch, A. y Navia, C, 2018) y dar el valor al diario de campo como una herramienta de investigación cualitativa, a través del cual se conoce de la fuente primaria, información para el desarrollo de la presente investigación.

Para el proceso de interpretación y análisis de los diarios se definieron tres etapas: en primer lugar, se hizo la lectura individual de cada uno de los 60 diarios de campo con el fin de seleccionar aquellos que evidenciaran mayor presencia de emociones, para lo cual se hizo necesario pasar de la lectura literal a la inferencial y determinar a través del lenguaje usado, aquellas expresiones que connotaron la vivencia de emociones respecto a la práctica pedagógica; posteriormente, se hizo análisis de contenido de la información seleccionada en el paso anterior, con el fin de ser agrupada según aproximación semántica en las narraciones. Por último, con la información concretada, se definieron las dos categorías de análisis: las emociones positivas y las emociones negativas las cuales fueron descritas con voces directas de los momentos experimentados por los maestros en formación, durante las prácticas pedagógicas.

\section{DISCUSIÓN Y RESULTADOS}

A partir del análisis de los diarios de campo se pudo identificar que los practicantes experimentaron, en su práctica pedagógica, emociones tanto positivas como negativas, que otorgan sentido y validez a su vocación docente. Entre las emociones positivas de mayor relevancia están, la felicidad, el placer y el amor, a través de las cuales se evidencia no solo el cumplimiento del deber asumido; sino que se compensa la dedicación, la responsabilidad y la creatividad para hacer de cada encuentro pedagógico una experiencia enriquecedora en el proceso de enseñanza y aprendizaje. Esto supone que maestro y estudiantes, satisfacen sus expectativas de formación y crecen mutua y empáticamente. Vale la pena destacar que la emoción positiva de mayor preponderancia fue el amor, considerada por Goleman (2018), como una de las siete emociones básicas experimentadas por los seres humanos en su interacción con el mundo, pero también en su interacción consigo mismo y con el alcance de las metas propuestas. 
Con respecto a las emociones negativas, las más recurrentes fueron el temor, la tristeza y la vergüenza. Estas tres representan las angustias, las incertidumbres y los agobios que antes, durante y después de su intervención pedagógica experimentaron los practicantes. El temor, vivenciado frente a lo desconocido, frente a la expectativa de lograr lo mejor; la tristeza, visible en algunas actitudes displicentes de los estudiantes en relación a las propuestas metodológicas; y la vergüenza al no haber cumplido con los compromisos asumidos como maestro que, aunque en formación, deben dar testimonio de su responsabilidad e idoneidad pedagógica. En fin, positivas o negativas las emociones, lograron movilizar las mentes, la creatividad, las energías, la toma de decisiones y hasta la vocación docente de muchos de los maestros en formación, que sin un espacio de práctica no hubiera sido posible comprender desde la teoría.

Hay que mencionar que, además de las emociones multivalentes que genera la práctica pedagógica, estas en "efecto dominó" conducen a procesos de autorreflexión y crítica sobre la misma; así como da apertura al mejoramiento continuo sobre cómo enseñar, cómo comprender las necesidades e intereses de los educandos y cómo responder a las tendencias sociales del mundo local y global, características propias de la praxis pedagógica y de la investigación educativa, esenciales para la consolidación de procesos educativos de mejor calidad. Por otro lado, se pudo comprender que, si bien es cierto la formación de maestros normalistas está bien cimentada en procesos pedagógicos y didácticos; a la hora de afrontar situaciones emergentes y espontáneas que se suscitan en el aula de clase, los practicantes se sienten abrumados, temerosos e inseguros frente a la toma de la mejor decisión que no lastime ni emocional, ni social, ni académicamente tanto a los estudiantes como así mismo en calidad de sujetos en formación, lo cual se relaciona con el aprender a ser y aprender a vivir juntos, dos de los pilares de la educación del siglo XXI (Delors, 1994).

Lo anterior, sitúa al maestro como un profesional que requiere no solo formación en el campo de la educación, sino en el campo de la inteligencia emocional, pues en el diario quehacer docente se vivencian situaciones revestidas de emociones, que en muchos momentos resultan contradictorias y que convergen en tensiones que desestiman el capital cognitivo y actitudinal del futuro profesional educativo. (Bedacarratz,2012). La formación de maestros además de ser pedagógica, debe ser en emociones no solo en relación a los valores, la ciudadanía y la ética, sino al constante movimiento de ideas, sentimientos y posturas que agiten el corazón de este profesional en potencia, pues mucho de lo que los practicantes trasladan a su experiencia como maestros en formación es lo que han recibido y percibido como estudiantes.

Finalmente, se pudo determinar que los maestros en formación, si hacen reflexión sobre su práctica y abordan asuntos de tipo pedagógico, metodológico, social y personal. Esta introspección les permite 
proponer y desarrollar acciones transformativas en su práctica pedagógica; aunque no siempre se produzcan los mejores resultados, por lo menos incitan a la praxis permanente de su quehacer; en este sentido, Chávez, et al (2021) en un estudio sobre los maestros normalistas plantean que:

"La reflexión sobre la práctica se hace de gran impacto para las futuras maestras, debido a que esta actividad reflexiva, dicen los docentes, la efectúan de manera sistemática, de forma que permite la retroalimentación de las actividades de las estudiantes y replantear su ejercicio docente" (p.12).

La reflexión de los normalistas superiores de la ENSI es registrada en los diarios de campo que, como instrumentos sistemáticos son textos abiertos que pueden leer y releer de manera íntima o compartida para avanzar en cambios personales y pedagógicos, porque como plantea Freire (1998) “el momento fundamental en la formación permanente de los profesores es el de la reflexión crítica sobre la práctica" (p.40).

Los actos pedagógicos desarrollados por los maestros, tanto dentro del aula como fuera de ella han de convertirse en asuntos de interés investigativo continuo, pues la práctica en sí misma no garantiza ni buenos procesos de enseñanza, ni significativos procesos de aprendizaje; estos llegan a consolidar su calidad y pertinencia en la medida en que sean investigados con el lento sistemático, riguroso, crítico y reflexivo del maestro, como una acción connatural de su desarrollo personal y profesional. Asuntos como las emociones del maestro en su rol pedagógico, merecen seguir siendo estudiados, pues la condición humana y sensible, determina en muchos casos, los desempeños profesorales del docente, a pesar de su formación académica.

Gráfica No. 1. Emociones latentes en el desarrollo de la práctica pedagógica de los maestros en formación 


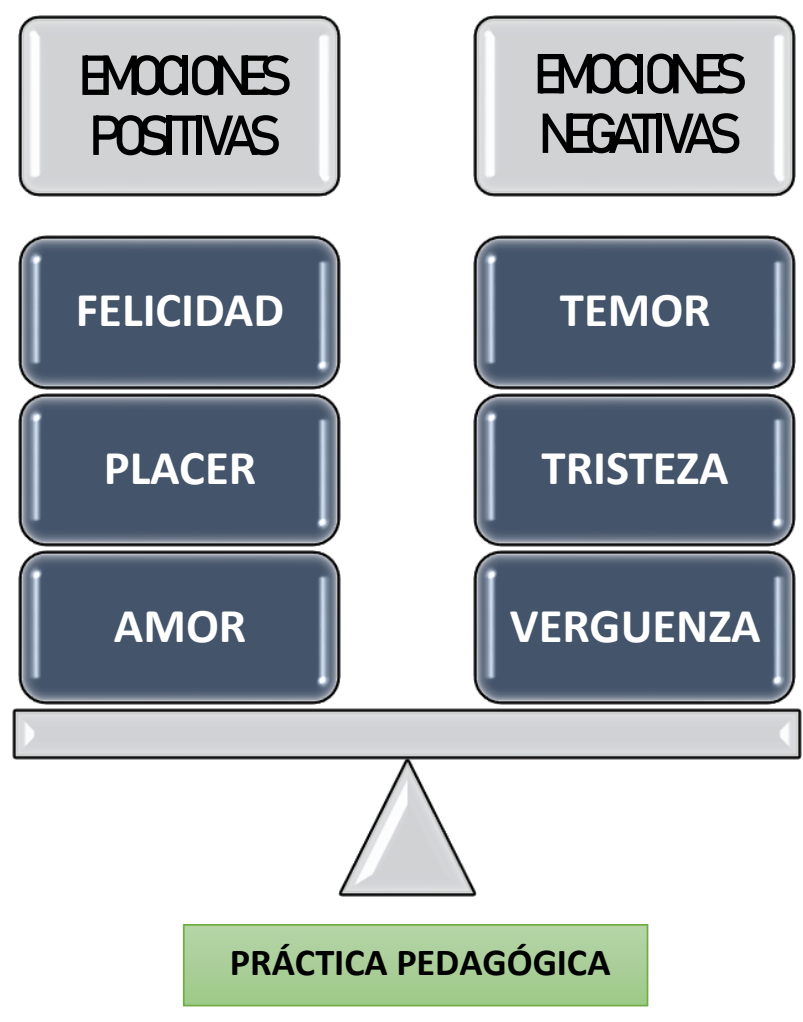

Fuente la autora

Esta gráfica representa la existencia de emociones en el desarrollo de la práctica pedagógica de los maestros en formación de la ENSI. Su presencia, inclina la balanza de acuerdo a las experiencias vividas en la cotidianidad del quehacer docente.

\section{CONCLUSIONES}

Luego del desarrollo del proceso de investigación se concluye que los maestros en formación valoran la práctica pedagógica, no solo porque es el escenario que les permite hacer trasposiciones didácticas y ejercer con autoridad metodológica su rol docente; sino porque en este escenario real se permiten interacciones con el conocimiento y con los estudiantes, que como sujetos cognoscentes y sociales viabilizan la formación del maestro de manera permanente. Estos encuentros generan en los practicantes múltiples emociones que los llevan a reafirmar su interés y vocación por la docencia, fruto de la reflexión permanente y crítica sobre su desempeño pedagógico.

Hablar de emociones en el contexto de la práctica pedagógica que forma maestros, es reconocer que estos no solo están ávidos de conocimientos pedagógicos, disciplinares, didácticos o normativos; sino que, como seres humanos los maestros también se emocionan con sus intervenciones, con sus propuestas y hasta con sus incertidumbres; todas estas se viven en la cotidianidad y llegan de manera inesperada al aula de clase, para lo cual muchos de los practicantes no están preparados porque no han sido formados en inteligencia emocional Goleman (2018), o en lo que ha llamado Gardner (2001) inteligencia 
intrapersonal e inteligencia interpersonal, saberes transversales que se deben hacer invisibles en los currículos y que están enfrentando de manera desprevenida a los maestros en formación, a la toma de decisiones que algunas veces resulta equivocada y logra desestabilizarlos emocionalmente. No se esperan encuentros escolares libres de dificultades; pero si se espera un maestro emocionalmente inteligente que sepa tomar decisiones oportunas y pertinentes según la necesidad y con la suficiente sensibilidad para comprender la situación y los sujetos involucrados en ella.

Por otro lado, se hace necesario reconocer la importancia del diario de campo como la herramienta pedagógica, a través de la cual se sistematiza el proceso de intervención educativa que desarrolla el maestro en su campo de práctica; a través del diario, el docente narra sus experiencias, describe sus percepciones y exterioriza sus emociones que poco a poco lo van constituyendo en un ser reflexivo y crítico, porque a partir de la escritura y la lectura de cada línea se plantean acciones transformadoras en procura de optimizar el proceso de enseñar y aprender, en el cual el maestro cumple una función dual. El diario de campo, se constituye, en una pieza clave para consolidar los procesos de praxis pedagógica, que a su vez se proyectan en intereses de investigación en el campo de las emociones que se generan desde la práctica pedagógica, por ejemplo; porque como plantea Goetz, Perun, Hall y Haag, 2006, citados por Paolini (2013) investigar en este campo demuestra que las experiencias emocionales están directamente relacionadas con el bienestar interno del estudiante; así mismo, la calidad de sus aprendizajes y de sus logros están directamente afectadas por las emociones; adicionalmente, la calidad de la comunicación entre estudiantes y maestros en el aula, así como los procesos de enseñanza están afectados por las emociones, los cuales deben ser reconocidas por los maestros para poder plantear acciones aliadas a estas y no que obstaculicen su intervención.

Finalmente, el desarrollo de este proceso investigativo develó que son muchos y variados los objetos de investigación sobre los cuales se puede indagar de manera sistemática y permanente en el campo de la formación de maestros, que no solo están conectados con el acto de enseñanza y aprendizaje propiamente dicho. Involucrar a diferentes actores en estos procesos (maestros en formación, maestros formadores, entre otros), permite delinear rutas de compromiso institucional para promover cultura investigativa, en instituciones como las Escuelas Normales, comprometidas con la formación de maestros empoderados de su rol pedagógico, social y sobre todo propositivo y transformador. 


\section{REFERENCIAS BIBLIOGRÁFICAS}

Bedacarratz, V (2012). Futuros maestros y construcción de una identidad profesional: una mirada psicosocial a los procesos que se ponen en juego en los trayectos de formación en la práctica. Santa Cruz, Argentina.

Chávez, F.P, Sáenz, F.A, Gutiérrez, C.M, De León, A.J (2021). South Florida Journal of Development, Miami, $\quad$ v.2, n.2, p.1 710-1723.Recuperado de: https://southfloridapublishing.com/ojs/index.php/jdev/article/view/270/269

Delors, J. (1996.): "Los cuatro pilares de la educación" en La educación encierra un tesoro: Informe a la UNESCO de la Comisión internacional sobre la educación para el siglo XXI. Madrid, España.

Domingo, R. A (2013). Práctica reflexiva para docentes. De la reflexión ocasional a la reflexión metodológica. Alemania: Publicia editorial.

Escuela Normal Superior de Ibagué (2011). Revista Panorama Normalista.

Freire. P (1998). Pedagogía de la autonomía. Siglo veintiuno de España editores, s.a.

Gardner, H (2001). Estructuras de la mente: la teoría de las inteligencias múltiples. Fondo de cultura económica. Bogotá, Colombia.

Goleman, D (2018). La inteligencia emocional. ¿Para qué las emociones? Bogotá DC: Colombia: Penguin Random House grupo editorial.

Hernández, S.R \& Otros (2014). Metodología de la investigación. México D.F: Mc Graw Hill interamericana editores, S.A DE C.V.

Hirsch, A. y Navia, C. (2018). Ética de la investigación y formadores de docentes. Revista Electrónica de Investigación Educativa, 20(3), 1-10.

Masi, A (2008). El concepto de praxis en Paulo Freire. En publicación: Paulo Freire. Contribuciones para la pedagogía. Moacir Godotti, Margarita Victoria Gómez, Jason Mafra, Anderson Fernández de Alencar (compiladores). CLACSO, Consejo Latinoamericano de Ciencias Sociales, Buenos Aires.

Ministerio de Educación Nacional (2008). Decreto 4790. Condiciones básicas de calidad del programa de formación complementaria de las escuelas normales superiores.

Ministerio de Educación Nacional (2015). Documento naturaleza y retos de las ENS.

Ministerio de Educación Nacional (2020). Decreto 1236. Organización y funcionamiento de las Escuelas Normales Superiores como instituciones educativas formadoras de docentes.

Paolini, P (2013). El papel de las emociones en los aprendizajes académicos. Cuestiones en Psicología educacional. Cuadernos de educación/01.F.Drago Andocopias. SL. La Laguna Tenerife.

Pekrun, R (2005). Progress and open problems in educational emotion research. Learning and instruction, 15, 497-506.

Porlán, R \& Martín J (2000). El diario del profesor. Sevilla: Diada editora. 